\title{
ICAM-1 Clustering on Endothelial Cells Recruits VCAM-1
}

\author{
Jaap D. van Buul, Jos van Rijssel, Floris P. J. van Alphen, Anna-Marieke van Stalborch, \\ Erik P. J. Mul, and Peter L. Hordijk
}

Department of Molecular Cell Biology, Sanquin Research and Landsteiner Laboratory, Academic Medical Center, University of Amsterdam, Plesmanlaan 125, 1066 CX Amsterdam, The Netherlands

Correspondence should be addressed to Jaap D. van Buul, j.vanbuul@sanquin.nl

Received 16 July 2009; Revised 12 November 2009; Accepted 20 December 2009

Academic Editor: Seamus J. Martin

Copyright (C 2010 Jaap D. van Buul et al. This is an open access article distributed under the Creative Commons Attribution License, which permits unrestricted use, distribution, and reproduction in any medium, provided the original work is properly cited.

In the initial stages of transendothelial migration, leukocytes use the endothelial integrin ligands ICAM-1 and VCAM-1 for strong adhesion. Upon adhesion of the leukocyte to endothelial ICAM-1, ICAM-1 is clustered and recruited to the adhered leukocyte, promoting strong adhesion. In this study, we provide evidence for the colocalization of VCAM-1 at sites of ICAM-1 clustering. Anti-ICAM-1 antibody-coated beads were used to selectively cluster and recruit ICAM-1 on primary human endothelial cells. In time, co-localization of ICAM-1 and VCAM-1 around the adherent beads was observed. Biochemical pull-down assays showed that ICAM-1 clustering induced its association to VCAM-1, suggesting a physical link between these two adhesion molecules. The association was partly dependent on lipid rafts as well as on F-actin and promoted adhesion. These data show that VCAM-1 can be recruited, in an integrin-independent fashion, to clustered ICAM-1 which may serve to promote ICAM-1-mediated leukocyte adhesion.

\section{Introduction}

Leukocytes migrate across endothelial monolayers to reach sites of inflammation. As a result of many intensive and detailed studies that have been carried out over the last 1015 years, it has become clear that the endothelium that lines the vessel wall is actively involved in supporting leukocyte transendothelial migration [1]. Upon binding of leukocytes to the endothelium, Ig-like cell adhesion molecules (CAMs) that are expressed on the apical surface of the endothelium, are clustered, recruited to sites of leukocyte adhesion, and concomitantly activated [2].

The main Ig-like CAMs on endothelium are InterCellular Adhesion Molecule-1 (ICAM-1/CD54) and Vascular Cell Adhesion Molecule-1 (VCAM-1/CD106). ICAM-1 is constitutively expressed at low levels on endothelial cells and 5- to 10 -fold up-regulated upon stimulation with inflammatory mediators such as $\operatorname{IL} 1 \beta$ and $\operatorname{TNF} \alpha[2,3]$. Leukocytes bind through their integrins LFA-1/Mac-1 to the extracellular domain of ICAM-1 [2]. Upon binding, ICAM-1 is clustered into membrane rafts [4], small, highly dynamic, steroland sphingo-lipid-enriched membrane rafts that can be stabilized to form large platforms through protein-protein interactions [5]. It has been reported that clustering of ICAM-1 induces intracellular signalling in endothelial cells, resulting in rearrangement of the actin cytoskeleton $[2,6,7]$. In line with this, binding of leukocytes through ICAM-1 induces a local increase in F-actin content at sites of adhesion [6].

VCAM-1 is weakly expressed on resting endothelium but is highly up-regulated upon stimulation with inflammatory mediators [2, 3]. Leukocytes adhere through the integrin VLA-4 to VCAM-1 on the endothelium. Similar as for ICAM-1, clustering of VCAM-1 induces intracellular signalling, leading to ROS production, p38 phosphorylation, and protein tyrosine phosphatase $1 \mathrm{~B}$ activation $[8,9]$. Barreiro and colleagues showed that the presence of the tetraspanins CD9 and CD151 is required for optimal adhesive functions of ICAM-1 and VCAM-1 [10]. Clearly, binding of leukocytes to either ICAM-1 or VCAM-1 induces multiple effects in the endothelium most of which serve to promote the interaction between the leukocyte and the endothelium as well as the subsequent transendothelial migration. 
Our data presented here indicate that VCAM-1 is recruited to sites of ICAM-1-induced adhesion independent of VCAM-1-binding to its cognate receptor; VLA-4. Biochemical studies showed that ICAM-1 clustering induces a physical association with VCAM-1. Using fluorescence microscopy, we demonstrate that VCAM-1 is recruited to clustered ICAM-1 in a time-dependent fashion. These data indicate that ICAM-1 and VCAM-1 co-operate to facilitate the adhesion and extravasation of leukocytes.

\section{Material and Methods}

2.1. Reagents and Antibodies. The following antibodies (Ab) were used: mAb against ICAM-1 (84H10); ICAM-1-FITC; VCAM-1 (1G11) from R\&D systems (Minneapolis, MN, USA); pAb against ICAM-1 (H-108) and VCAM-1 (C19) from Santa Cruz Biotechnology (Heerhugowaard, The Netherlands); mAb against PECAM-1 (CD31) and isotype control IgG from Sanquin Immunoreagents (Amsterdam, The Netherlands); Alexa488-labelled $c \alpha \mathrm{m}$, Alexa488 c $\alpha \mathrm{r}$; Alexa-594 $\mathrm{c} \alpha \mathrm{m}$; Alexa-594 $\mathrm{c} \alpha \mathrm{r}$ and Alexa647 $\mathrm{c} \alpha \mathrm{g}$ from Invitrogen (Leiden, The Netherlands). For actin staining Bodipy650/665 phalloidin and Texas Red-X phalloidin were used (Invitrogen).

2.2. Cell Cultures. HUVECs were obtained from Cambrex (East Rutherford, NJ) and cultured as described previously in in [11]. Endothelial cells were activated with $10 \mathrm{ng} / \mathrm{mL}$ TNF $\alpha$ (R\&D systems) overnight, to mimic inflammation and grown to confluent monolayers over at least 3 days. HeLa cells were routinely cultured in IMDM supplemented with $10 \%$ FCS and $5 \%$ glutamine. All cell lines were cultured or incubated at $37^{\circ} \mathrm{C}$ at $5 \% \mathrm{CO}_{2}$.

2.3. Immunofluorescent Imaging. HUVECs were cultured on FN-coated glass coverslips, fixed, and immunostained as described in [11] and stained for proteins as indicated, followed by secondary fluorescently labelled Abs. Images were recorded with a ZEISS LSM510-Meta confocal microscope (Carl Zeiss, Jena, Germany) with appropriate filter settings. Cross-talk between the green and red channel was avoided by use of sequential scanning.

2.4. Coating of Beads. Magnetic g $\alpha \mathrm{m}$ IgG Ab-coated Dynabeads (Invitrogen) were coated with ICAM-1, VCAM-1 or IgG isotype control mAbs according to the manufacturers' protocol. Polystyrene $10 \mu \mathrm{m}$ and $3 \mu \mathrm{m}$ beads (Polysciences Inc., Eppelheim, Germany) were coated with antibody according to the manufacturer's instructions and used for immunofluorescent imaging.

2.5. Adhesion of Beads. HeLa cells were cultured on FNcoated glass coverslips and transfected with ICAM-1mCherry or VCAM-1-GFP or a combination. Next, ICAM1 coated beads were allowed to adhere to the cells for 30 minutes at $37^{\circ} \mathrm{C}$ at $5 \% \mathrm{CO}_{2}$, after which the cells were put on ice and gently washed three times with ice-cold PBS, containing $1 \mathrm{mM} \mathrm{CaCl} 2$ and $\mathrm{MgCl}_{2}$. Then, samples were fixed and immunostained for F-actin as described in [11]. Beads that adhered to positive transfected cells were counted.

2.6. Protein Crosslinking. Crosslinking experiments were carried out as described in [11]. Briefly, HUVEC were treated with mouse-antihuman antibody against a protein as indicated for 30 minutes, washed to remove all the nonbound Abs and subsequently incubated for an additional 30 minutes with goat-antimouse IgG antibodies that crosslink mouse-antihuman Abs through their Fc-tail. This results in clustering of the protein of interest.

2.7. Immunoprecipitation and Western Blot Analysis. Cells were grown to confluency on FN-coated dishes $\left(50 \mathrm{~cm}^{2}\right)$, washed twice gently with ice-cold $\mathrm{Ca}^{2+}$ and $\mathrm{Mg}^{2+}$ containing PBS, and lysed in $300 \mu \mathrm{L}$ of lysis buffer $(25 \mathrm{mM}$ Tris, $150 \mathrm{mM} \mathrm{NaCl}, 10 \mathrm{mM} \mathrm{MgCl}$, 2 mM EDTA, 1\% Triton $\mathrm{X}-100$, with the addition of fresh protease-inhibitor-cocktail tablets (Boehringer) pH 7.4). After 10 minutes on ice, cell lysates were collected and precleared for 30 minutes at $4^{\circ} \mathrm{C}$ with Protein-G Sepharose beads (GE Healthcare, Sweden; $15 \mu \mathrm{L}$ for each sample). The supernatant, cleared by centrifugation $\left(14.000 * \mathrm{~g}, 5\right.$ minutes at $4^{\circ} \mathrm{C}$ ) was incubated with $15 \mu$ l protein Sepharose beads that were coated with the $\mathrm{Ab}$ of interest for 1 hour at $4^{\circ} \mathrm{C}$ under continuous mixing. The beads were washed 3 times in lysis buffer and proteins were eluted by boiling in SDS-sample buffer containing 4\% 2 -mercaptoethanol. The samples were analyzed by $10 \%$ SDSPAGE. Proteins were transferred to $0.45 \mu \mathrm{m}$ nitro-cellulose (Schleicher and Schnell Inc., NH) and the blots were first blocked with $5 \%(\mathrm{w} / \mathrm{v})$ low-fat milk in Tris-Buffered Saline Tween-20 (TBST) for 1 hour, subsequently incubated at room temperature with the appropriate Abs for 1 hour, followed by 30 minutes incubation with HRP-conjugated secondary Abs at RT. Between the various incubation steps, the blots were washed 5 times with TBST and finally developed with an enhanced chemiluminescence (ECL) detection system (Amersham).

2.8. Statistics. Students $T$-test was used to calculate statistical significance.

\section{Results}

Upon inflammation, not only the expression of ICAM-1 but also of VCAM-1 is increased, up to 20 -fold [2, 3]. ICAM-1 and VCAM-1 hardly colocalize with each other on TNF $\alpha$ treated endothelial cells (Figure 1(a)). ICAM-1 localizes more to the periphery of the cell, and VCAM-1 staining was more pronounced at the cell-body (Figure 1(a)). Detailed analysis showed however that VCAM-1 did co-localize to some extent with ICAM-1 in microvilli, but we found no colocalization at the baso-lateral side of the cells (Figure 1(b)). To address leukocyte-induced coclustering of ICAM-1 and VCAM-1, we made use of HL60 cells, which were differentiated towards a neutrophil-like, myeloid-lineage cell type 

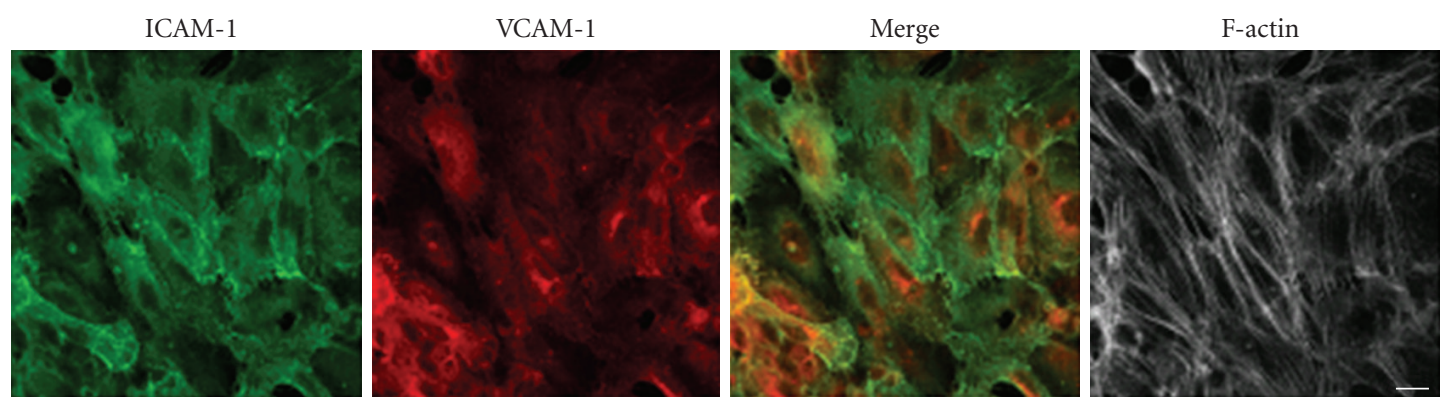

(a)
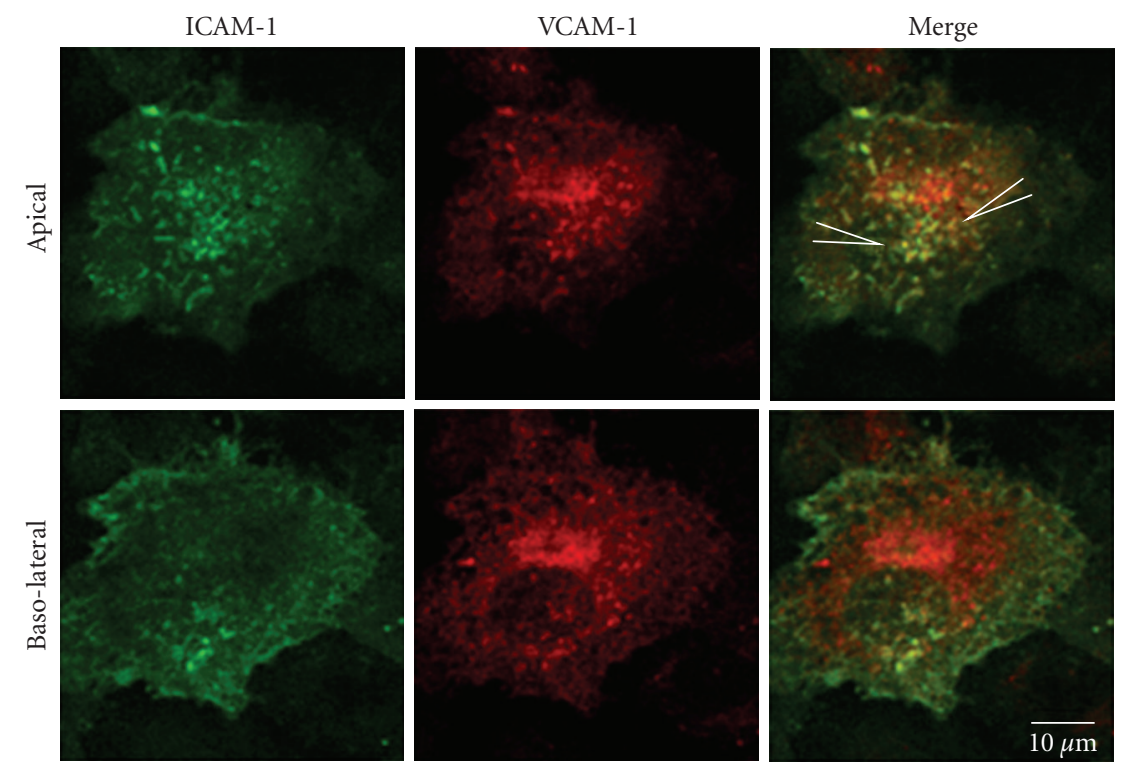

(b)
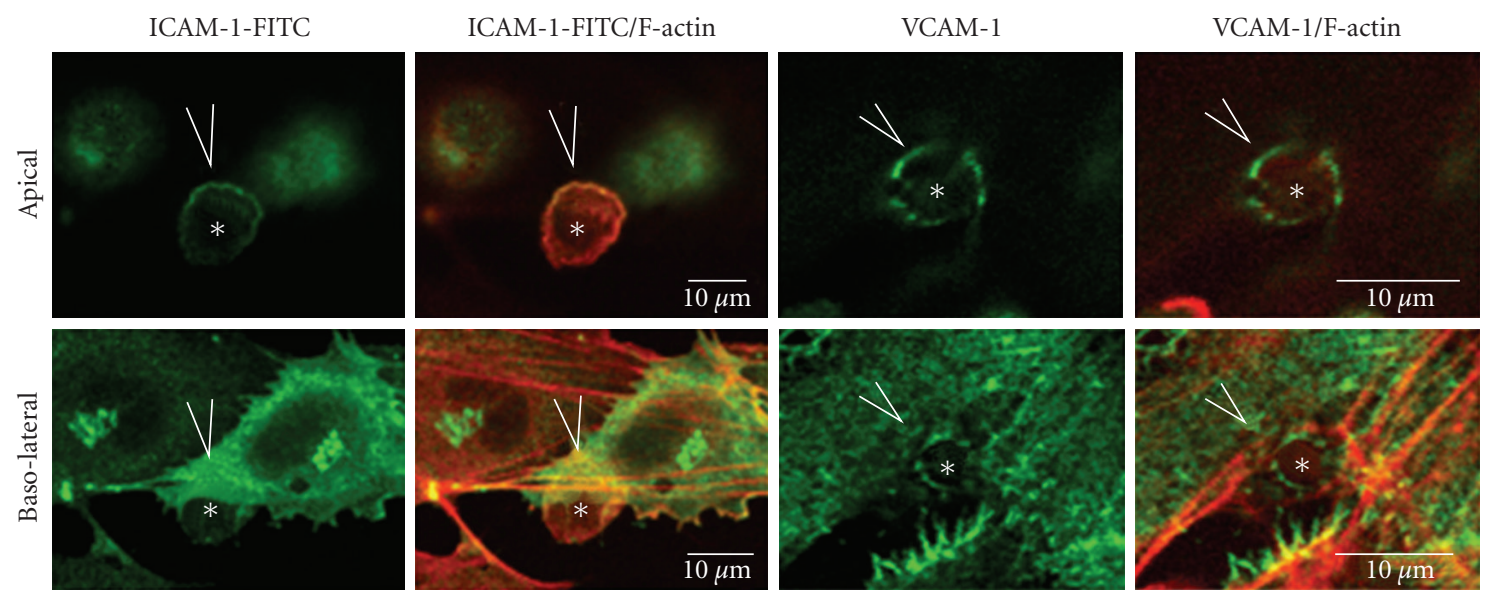

(c)

Figure 1: ICAM-1 and VCAM-1 localization in TNF $\alpha$-stimulated endothelial cells. (a) TNF $\alpha$-treated HUVECs were fixed and stained as indicated. Bar, $20 \mu \mathrm{m}$. (b) Detailed analysis of ICAM-1 and VCAM-1 co-localization at the apical surface (arrowheads) in TNF $\alpha$-treated endothelial cells that were fixed and stained as indicated. Bar, $10 \mu \mathrm{m}$. (c) Differentiated HL60 cells were allowed to adhere to TNF $\alpha$ treated HUVECs for 30 minutes, after which cells were fixed and stained as indicated. Apical focal plane shows that ICAM-1 and VCAM-1 (arrowheads) are recruited around adhered HL60 cells (asterisks). Bar, $10 \mu \mathrm{m}$. 
using 1.3\% DMSO. These cells express all required integrins to bind to ICAM-1 or VCAM-1, that is, LFA-1, Mac-1, and VLA-4 (data not shown). Following seeding of these cells on activated endothelial cells, we found that both adhesion molecules were recruited around firmly adherent leukocytes, as was also described by Barreiro et al. [12] (Figure 1(c)). The clustering of ICAM-1 and VCAM-1, induced by HL60 cells, was also observed in human microvascular endothelial cells (data not shown).

In order to specifically focus on ICAM-1-mediated adhesion, we used anti-ICAM-1 antibody-coated beads, hereafter called ICAM-1 beads that induce clustering of ICAM- 1 on the surface of TNF $\alpha$-treated endothelial cells. Within 10 minutes, endogenous ICAM-1 was recruited to sites of bead adhesion, as determined by confocal microscopy using FITC-conjugated ICAM-1 antibodies (Figure 2(a)). As a negative control, anti-MHC class I-antibody coated beads or anti-IgG antibody coated beads were used. No recruitment of ICAM-1 could be observed using these beads (data not shown). Costaining for VCAM-1 showed that, in time, VCAM-1 also was recruited to adhesion sites that were induced by ICAM-1 beads, although no VCAM-1 binding antibodies were present (Figure 2(a)). Detailed $X-Z$ analysis showed that ICAM-1 and VCAM-1 are both accumulating in the membrane structure around adhered ICAM-1 beads (see Figure lin Supplementary Material available online at doi:10.1155/2010/120328 ). Quantification of VCAM1-recruitment to membrane around these anti-ICAM-1antibody coated beads showed that at 60 minutes, more than $80 \%$ of the beads recruited, next to ICAM-1, also VCAM-1 (Figure 2(b)). Additional experiments in which the time course was extended to 120 minutes showed that the percentage of co-localization between ICAM-1 and VCAM1 around the beads did not change. Conversely, using antiVCAM-1-antibody coated beads, hereafter called VCAM-1 beads, seeded on TNF $\alpha$-treated endothelial cells we could demonstrate that after 60 minutes, ICAM-1 was recruited to clustered VCAM-1 (Figure 2(c)). These data suggest that ICAM-1 and VCAM-1 associate upon clustering of either integrin ligand.

To investigate this further, we used ICAM-1-antibody coated magnetic beads to cluster ICAM-1 on the surface of the endothelium, followed by a pull-down assay from the cell lysates. Interestingly, these experiments showed that VCAM-1 associated to the ICAM-1 beads (Figure 3(a)). As controls, we show that VCAM-1 beads efficiently precipitated VCAM-1 from endothelial cell lysates; whereas anti-IgG1antibody coated beads were unable to precipitate VCAM1 (Figure 3(a)). Vice versa, endogenous ICAM-1 was precipitated with VCAM-1 beads from endothelial cell lysates (Figure 3(b)), albeit less efficient than the pull-down using ICAM-1 beads (compare with Figure 3(a)). As a control, we used anti-IgG1-antibody coated beads which did not precipitate ICAM-1 (Figure 3(b)). To study if clustering of ICAM-1 promotes the association with VCAM-1, ICAM1 antibodies in solution were used to crosslink ICAM1 on the surface of the endothelium. This method has been used extensively by many groups including ours $[2$, $4,7,11]$. Subsequently, the cells were lysed and ICAM-1 was immunoprecipitated from the cell lysate. The results, presented in Figure 3(c), showed that VCAM-1 association with ICAM-1 is stimulated when ICAM-1 is clustered, that is, upon crosslinking. ICAM-1 immunoprecipitation following cross-linking of MHC-class I molecules did not promote association of VCAM-1 (Figure 3(c)). To show that ICAM- 1 is specifically precipitated by ICAM-1 beads and not by anti-MHC class I-antibody coated beads, pull-down assays were performed and analyzed for the presence of ICAM-1. The results showed that ICAM-1- but not antiMHC-antibody coated beads precipitated ICAM-1 from endothelial cell lysates (Figure 3(d)). In addition, antiPECAM-1-antibody coated beads, which cluster of PECAM1, did not precipitate ICAM-1 (Figure 3(e)). These data indicate that ICAM-1 and VCAM-1 physically associate upon clustering.

Previous literature suggests that ICAM-1 shifts to lipidrafts upon clustering [4]. To investigate if the association between ICAM-1 and VCAM-1 depends on lipid raft formation, $\mathrm{TNF} \alpha$-stimulated endothelial cells were pretreated with the cholesterol chelator cyclodextrin (CD). Using ICAM1 beads on endothelial cells, the recruitment of VCAM1 after 60 minutes of adhesion showed a reduction of approximately $30 \%$ of VCAM-1 recruitment to ICAM1-positive adhesive sites (Figure 4(a)). To study if actin polymerization is required for the recruitment of VCAM1 to ICAM-1 beads, endothelial cells were pre-treated with Cytochalasin B. Quantification showed that after 60 minutes, VCAM-1 was less efficiently recruited to ICAM1 beads when actin polymerization was blocked. Thus, actin polymerization is required for optimal recruitment of VCAM-1 to ICAM-1-induced adhesion sites (Figure 4(a)). To study if VCAM-1 had an additional effect on ICAM1-induced adhesion sites, ICAM-1 beads were allowed to adhere for 60 minutes to HeLa cells that were transfected with GFP, ICAM-1-mCherry, VCAM-1-GFP, or doubletransfected with ICAM-1-mCherry and VCAM-1-GFP. The results showed that ICAM-1 beads efficiently adhered to ICAM-1-mCherry expressing cells $(2 \pm 0.5$ ICAM- 1 beads per ICAM-1-mCherry-positive cell) but not to VCAM-1GFP or GFP expressing cells (Figure 4(b)). Interestingly, a significant increase in adhesion of ICAM-1 beads to the cells was measured when ICAM-1 and VCAM-1 were both expressed $(3.25 \pm 0.7$ ICAM- 1 beads per ICAM-1-mCherrypositive cell) (Figure 4(b)). Using VCAM-1 beads, similar results were obtained as for the ICAM-1 beads (Figure 4(c)). In addition, non-differentiated HL60 cells that express (in contrast to differentiated HL60 cells) primarily LFA-1, the counter-receptor for ICAM-1, and low levels of beta-1 integrin, and U937 cells that express primarily VLA-4, the counter-receptor for VCAM-1 were used in the same setup as described for Figures 4(b) and 4(c). Interestingly, also the adhesion of these cells to double-transfected cells was increased (Figure 4(d)). These data suggest that initial leukocyte adhesion may be mediated by a single adhesion molecule (e.g., ICAM-1), but that in time, when the adhesion sites mature, other transmembrane adhesion molecules such as VCAM-1 are coclustered and assisted ICAM-1 in its adhesive function. 


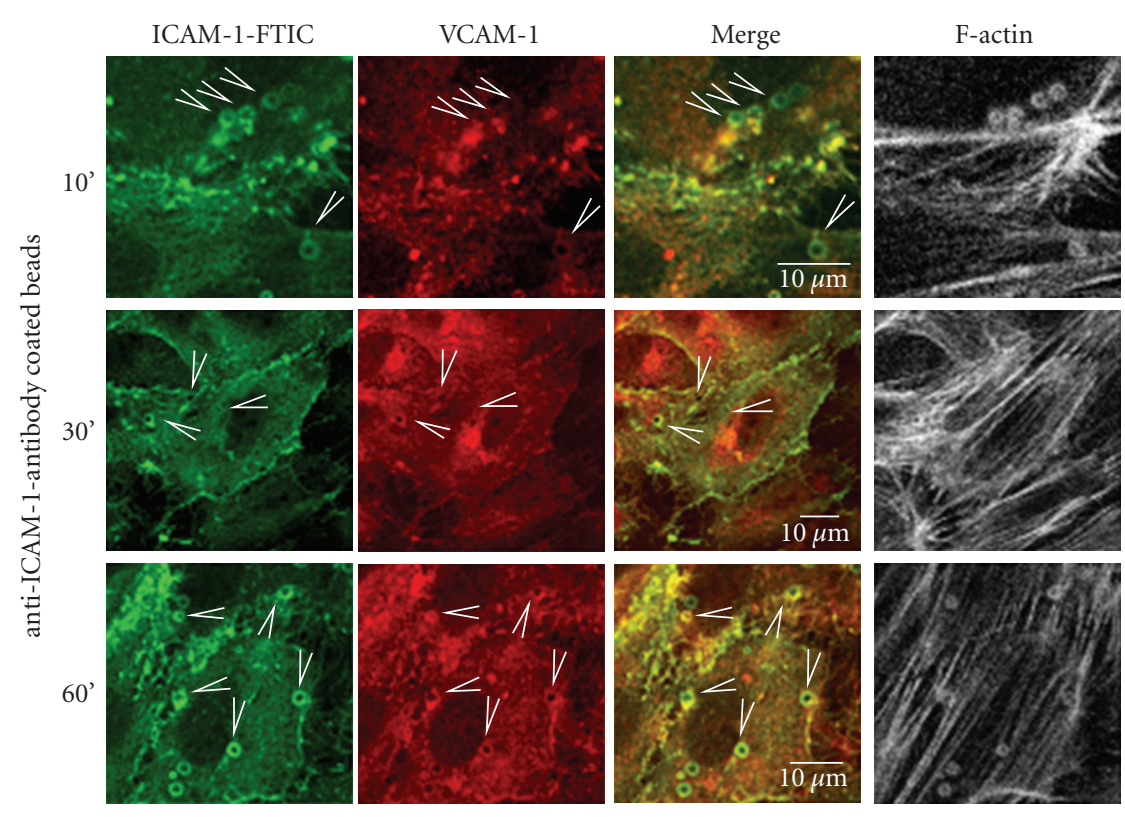

(a)

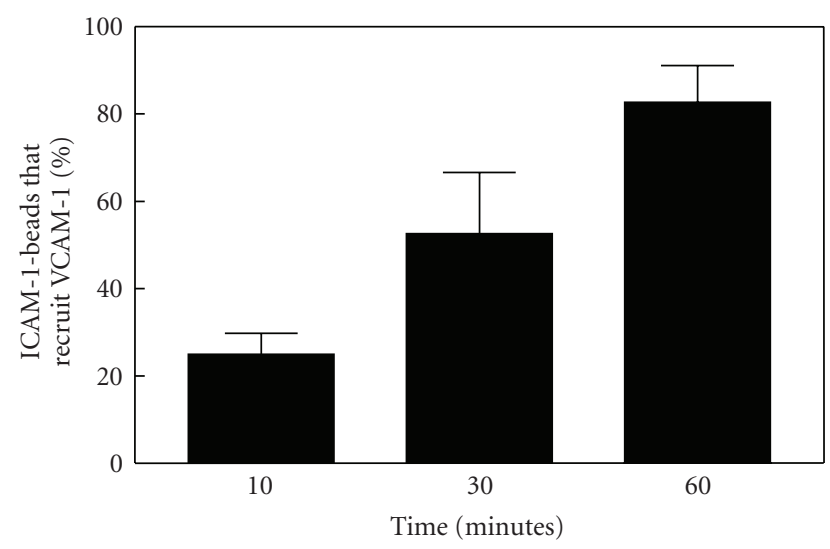

(b)
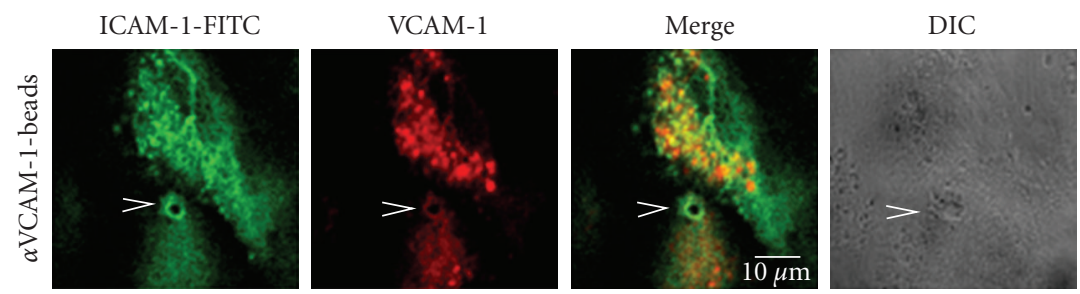

(c)

FIGURE 2: VCAM-1 is recruited to anti-ICAM-1-antibody coated beads. (a) ICAM-1 beads (diameter $3 \mu \mathrm{m}$ ) were allowed to adhere to TNF $\alpha$ treated HUVECs. Subsequently, the samples were fixed at the indicated time points and stained as indicated. VCAM-1 is recruited to ICAM1 beads (arrowheads) in time. Bar, $10 \mu \mathrm{m}$. (b) Quantification of VCAM-1 recruitment to ICAM-1-induced adhesion sites. The number of adhered ICAM-1-antibody beads that were positive for ICAM-1-FITC staining was set as 100\% and percentage of beads that co-localized with VCAM-1 was counted. At 60 minutes adhesion, approximately $80 \%$ of ICAM-1 beads that recruited ICAM-1 also recruited VCAM-1. Data are mean \pm SEM of three independent experiments. (c) VCAM-1 beads (diameter $3 \mu \mathrm{m}$ ) were allowed to adhere to TNF $\alpha$-treated HUVECs and stained as indicated. ICAM-1 is recruited to VCAM-1 beads (arrowheads). Bar, $10 \mu \mathrm{m}$. 


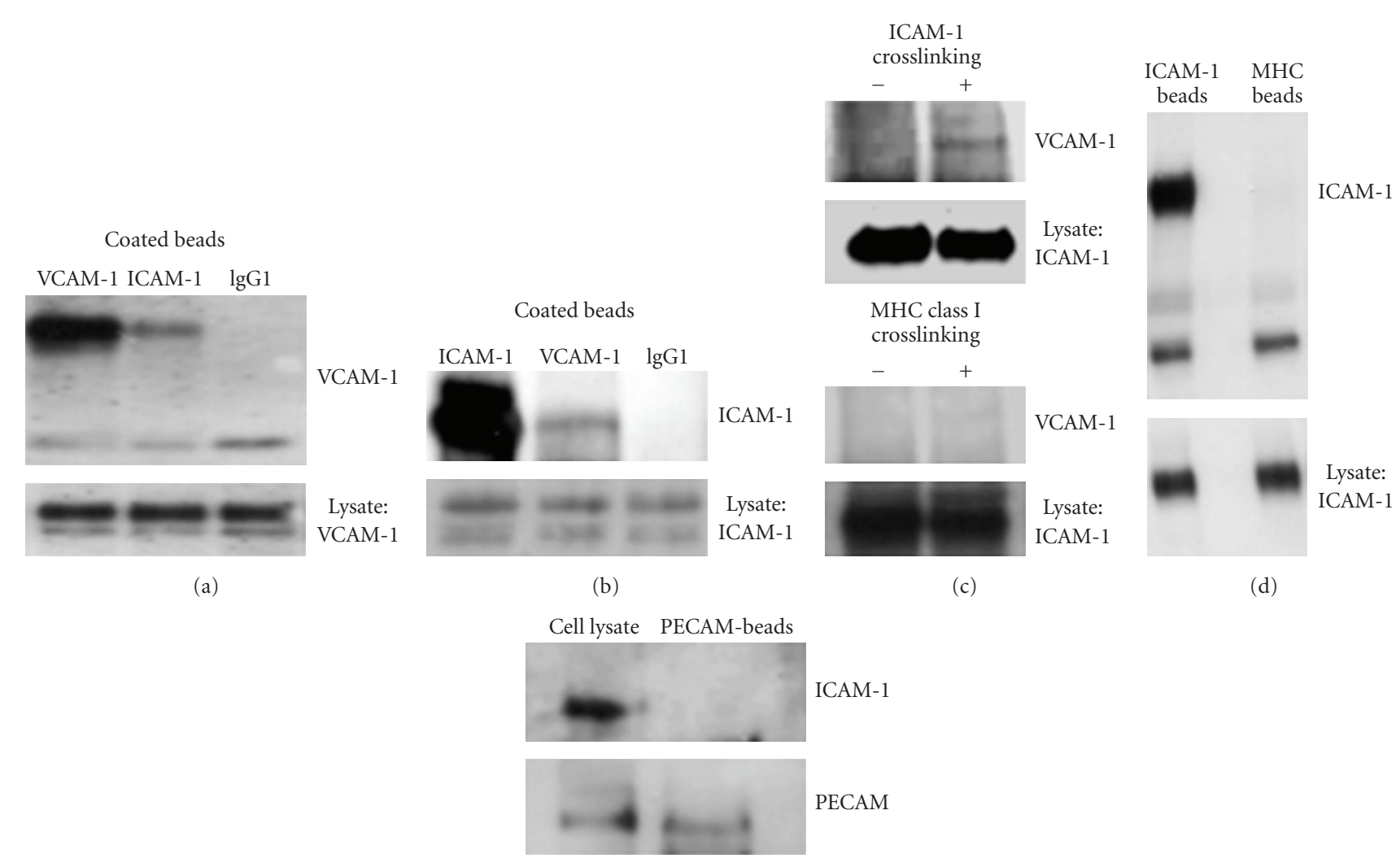

(e)

FIGURE 3: VCAM-1 associates with clustered ICAM-1. (a) Beads were coated with the indicated Abs as described in Section 2 and subsequently incubated with lysates from TNF $\alpha$-treated HUVECs, followed by centrifugation, washing and analysis of bound proteins by SDS-PAGE and Western blotting. Blots were stained for VCAM-1 and show that VCAM-1 was precipitated with VCAM-1 beads and also with ICAM-1 beads. No VCAM-1 was detected in pull-downs using anti-IgG antibody coated beads. Lower panel shows expression of VCAM-1 in cell lysates as a loading control. Data are representative for three experiments. (b) The pull-down experiment was carried out as described under (a) and blots were stained for ICAM-1. ICAM-1 was precipitated with ICAM-1 beads, and a fraction of ICAM-1 associates with VCAM-1 beads, albeit less efficient than the reciprocal experiment shown in (a). No ICAM-1 was detected in pull-down experiments using anti-IgG coated beads. Lower panel shows expression of ICAM-1 as loading control in cell lysates. Data are representative for three experiments. (c) TNF $\alpha$-stimulated HUVECs were incubated with ICAM-1 Ab in solution for 30 minutes and subsequently crosslinked with secondary antimouse $\mathrm{Ab}$ for an additional 30 minutes. Cells were lysed and ICAM-1 was immunoprecipitated and analyzed by Western blotting, which shows that ICAM-1-clustering induced association with VCAM-1. Lower panel shows ICAM-1 expression in cell lysates. Bottom panel shows that crosslinking of MHC class I protein did not induce any binding of VCAM-1. (d) ICAM-1 is precipitated by ICAM-1 beads, but not by anti-MHC class I antibody coated beads. Lower panel shows expression of ICAM-1 in cell lysates. (e) No ICAM-1 is precipitated by anti-PECAM-1 antibody coated beads (upper panel). Lower panel shows precipitation of PECAM-1 with the beads.

\section{Discussion}

Members of the Ig-like superfamily of cell adhesion molecules such as ICAM-1 and VCAM-1 are key players in the process of inflammation. Upon stimulation with inflammatory cytokines such as TNF $\alpha$ and IL1 $\beta$, these adhesion molecules are highly upregulated and support the migration of leukocytes to sites of infection or inflammation. Both adhesion molecules have specific counter-receptors: ICAM-1 binds the integrins LFA-1 and Mac-1, expressed on most leukocytes and VCAM-1 interacts with VLA-4, expressed on monocytes. It is believed that these interactions can occur in parallel. Our group has shown that blocking either ICAM-1 or VCAM-1 with antibodies reduces the migration of hematopoietic stem cells across endothelial monolayers in [11]. Blocking both molecules decreased stem cell transmigration in an additive fashion in [11]. In the present study, we show that ICAM-1 clustering recruits VCAM-1 and vice versa, independent from their cognate receptor and in a time-dependent fashion.

Additionally, we show that this recruitment partially depends on the presence of lipid-rafts that have previously been implicated in adhesion receptor clustering [4] and promote ICAM-1-adhesive function. Our data suggest that the clustering of transmembrane adhesion molecules by adherent leukocytes provides a platform recruiting other molecules to form a multiprotein complex that will assist leukocytes to adhere to and migrate across the endothelial monolayer. However, Ig-like CAM CD31/PECAM-1 appears not to participate in the formation of these platforms, at least 


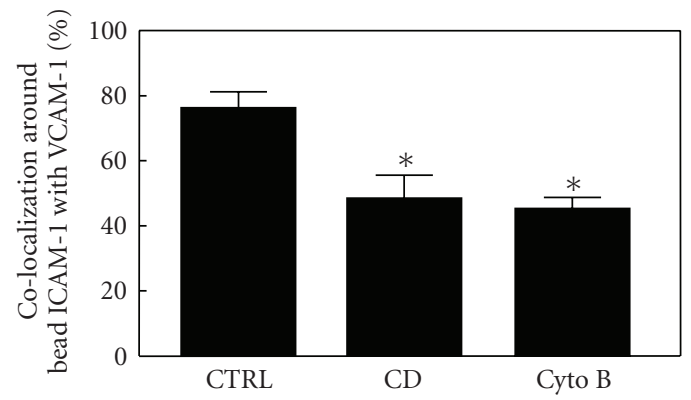

(a)

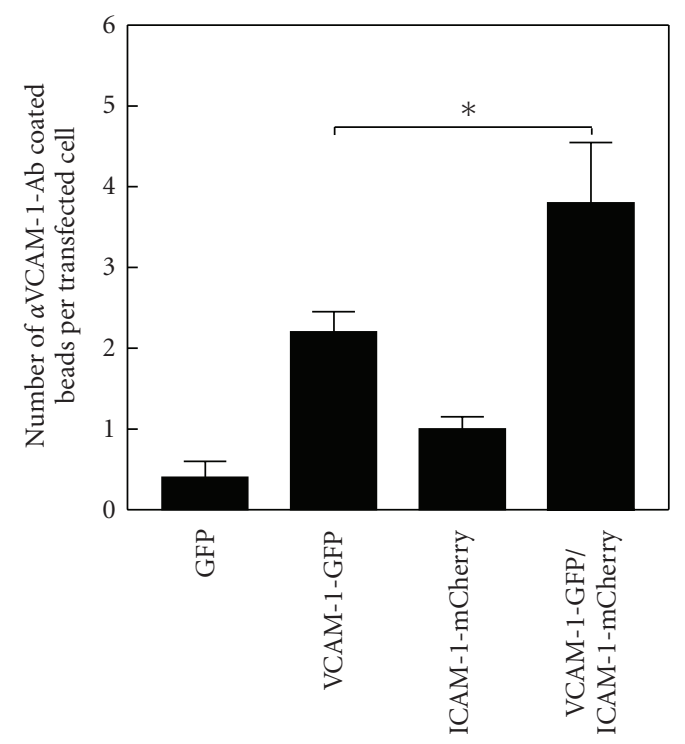

(c)

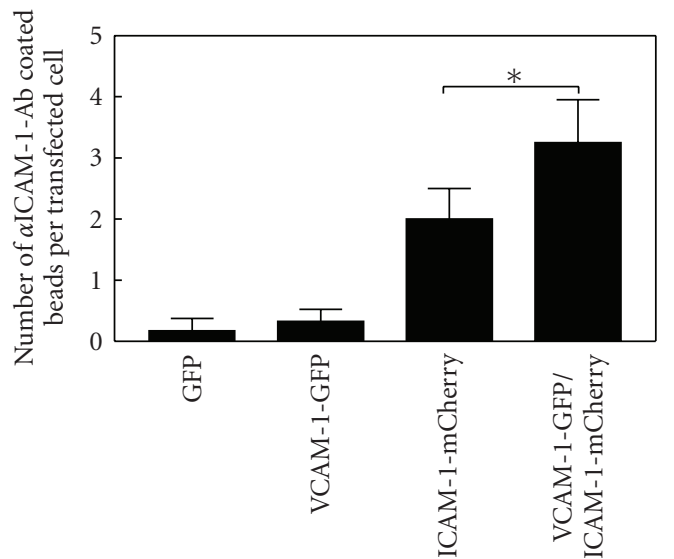

(b)

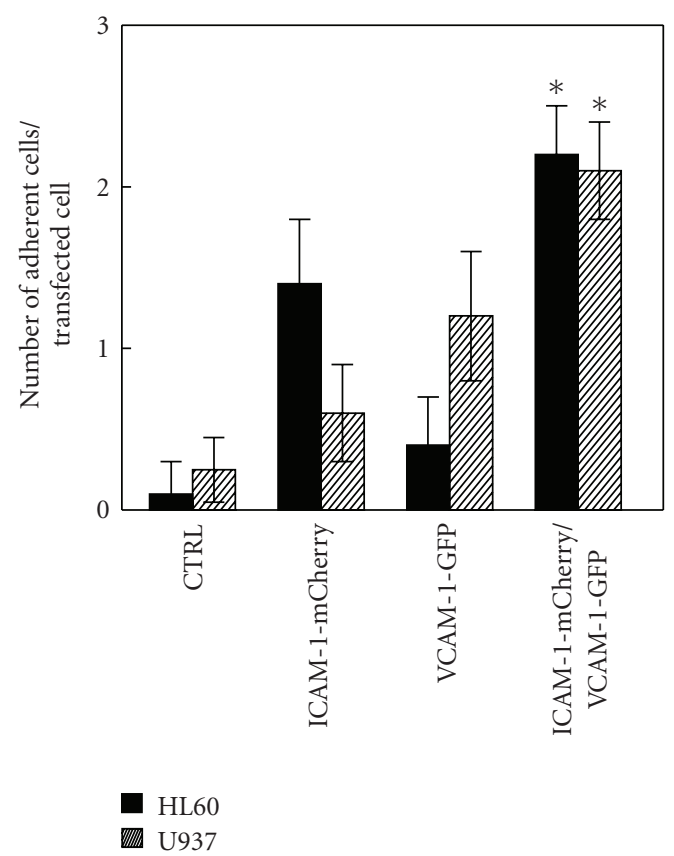

(d)

FIGURE 4: ICAM-1-induced VCAM-1 recruitment depends on lipid-rafts and actin polymerization and promotes ICAM-1-induced adhesion. (a) Anti-ICAM-1-antibody coated beads were allowed to adhere for 60 minutes to TNF $\alpha$-stimulated HUVECs that were left untreated (CTRL) or were pretreated for 30 minutes with either cyclodextrin $(\mathrm{CD} ; 3.8 \mu \mathrm{M})$ or cytochalasin B (CytoB; $1 \mu \mathrm{g} / \mathrm{mL})$. Subsequently, cells were fixed and immunostained with ICAM-1-FITC and VCAM-1 Abs. VCAM-1 recruitment to ICAM-1 beads was quantified which showed that close to $80 \%$ of ICAM-1 beads which recruited ICAM-1 (set as 100\%) also recruited VCAM-1. CD pretreatment reduced VCAM-1 recruitment with $40 \%$ and CytoB with 50\%. Experiment was carried out three times. Data are mean \pm SEM. ${ }^{*} P<.05$. (b) Anti-ICAM-1-antibody coated beads were allowed to adhere for 60 minutes to HeLa cells that were transfected as indicated. Subsequently, cells were fixed and analyzed by confocal microscopy for number of ICAM-1 beads that adhered to one transfected cell. 50 transfected cells per experiment were analyzed and the experiment is carried out in duplicate. The experiment was carried out three times. Data are mean \pm SEM. ${ }^{*} P<.05$. (c) AntiVCAM-1-antibody coated beads were allowed to adhere for 60 minutes to HeLa cells that were transfected as indicated. Subsequently, cells were fixed and analyzed by confocal microscopy for number of VCAM-1 beads that adhered to one transfected cell. 50 transfected cells per experiment were analyzed and the experiment is carried out in duplicate. The experiment was carried out three times. Data are mean \pm SEM. ${ }^{*} P<.05$. (d) Nondifferentiated HL60 or U937 cells were allowed to adhere for 60 minutes to HeLa cells that were transfected as indicated. Subsequently, cells were fixed and analyzed by confocal microscopy for number of VCAM-1 beads that adhered to one transfected cell. 50 transfected cells per experiment were analyzed and the experiment is carried out in duplicate. The experiment was carried out two times. Data are mean \pm SEM. ${ }^{*} P<.05$. 
not in those induced by ICAM-1-VCAM-1, as we did not find any detectable PECAM-1 in the ICAM-1- or VCAM-1-based pull-downs.

ERM-proteins (i.e., ezrin, radixin, and moesin) are adaptor proteins that bind the actin cytoskeleton and have been found to associate to either ICAM-1 or VCAM-1 [12]. However, we have not found an interaction between one of the ERM-proteins and the intercellular tail of ICAM-1 or VCAM-1 (personal observation JDvB and JvR). Therefore, the ERM-family proteins are most likely not the intermediate adapter proteins that link ICAM-1 to VCAM-1. Interestingly, ICAM-1 intracellular tail contains two SH3-type domains; whereas the VCAM-1 intracellular tail harbours a PDZbinding domain at its very end [13]. Therefore, a protein that is rich in prolines and also carries a PDZ domain may be a candidate to link ICAM-1 and VCAM-1 directly.

It is appreciated that ICAM-1 recruits several intracellular signalling and adaptor proteins upon clustering (see for review $[2,6])$. Nevertheless, to our knowledge, it has never been shown that ICAM-1 can actively recruit other transmembrane adhesion molecule family members upon clustering. The previous papers have reported that neuronal(N-) CAM, a family member of the endothelial CAMs, recruits FGFR-4 and N-cadherin in L-cells [14]. These authors also showed that the signalling molecule Src-kinase and the actin-linker protein cortactin are present in the same complex with N-CAM. Src and cortactin are also found in relation to ICAM-1 function: antibody-induced crosslinking of ICAM-1 or clustering of ICAM-1 using anti-ICAM-1antibody coated beads induces the activation of Src [1517]. Along this line, ICAM-1 may serve, like N-CAM, as a docking site for proteins, not only intracellular but also transmembrane proteins.

Interestingly, Barreiro and coworkers recently reported the existence of so-called "endothelial adhesive platforms" (EAPs). These platforms are composed of small, tetraspaninenriched microdomains that express ICAM-1 and VCAM1 [18]. The clustering of ICAM-1 and VCAM-1 in these EAPs is independent of integrin engagement and the actin cytoskeleton. Our data are in line with the report by Barriero and coworkers and show that ICAM-1 and VCAM-1 Colocalize upon clustering of these proteins by antibody-coated beads. Thus, concentrating either ICAM-1 or VCAM-1 may lead to a clustering of nearby EAPs, resulting in further increasing the local concentration of ICAM-1 and VCAM-1 at these sites.

A platform for protein complexes is an efficient way to control and merge several signalling pathways [18-20]. These protein platforms involve tetraspanins: small palmitoylated polypeptides that span the plasma membrane four times $[18,21,22]$. Recently, it has been published that tetraspanins play an important role in the function of the transmembrane proteins ICAM-1 and VCAM-1 [18]. Other work from Barreiro and CO-workers shows that reduction of expression levels of the tetraspanins CD9 and CD151 significantly reduces the adhesion capacities of ICAM-1 and VCAM1 [10]. Moreover, blocking antibodies against endothelial tetraspanin CD81 decreased the migration of monocytes across endothelial monolayers [23]. These data indicate that the formation of a tetraspanin-rich protein platform is crucial for adhesion function and proper leukocyte crossing. Our data indicated that coexpression of VCAM1 in ICAM-1-expressing monolayers did promote adhesion of leukocytes. However, no significant increase in neutrophil transmigration under static conditions using HeLa cells that expressed both ICAM-1 and VCAM-1 was measured, compared to HeLa cells that only expressed ICAM-1-GFP (data not shown).

Together, these results underscore the complexity of the molecular events that accompany leukocyte adhesion and transendothelial migration. Defining the requirements within the leukocytes and the endothelium, most likely involving a combination of outside-in and inside-out signalling, warrant further research.

\section{Acknowledgments}

The authors would like to thank Dr. F. Sanchez-Madrid for providing the cDNAs encoding ICAM-1-GFP and VCAM1-GFP. This work was supported by the Dutch Heart Foundation (Grant no. 2003B012); The first and the third author are supported by the Dutch Heart Foundation (grant no. 2005T039) and NWO Veni grant 916.76.053. The second author was supported by the Academic Medical Center, Amsterdam.

\section{References}

[1] T. A. Springer, "Traffic signals for lymphocyte recirculation and leukocyte emigration: the multistep paradigm," Cell, vol. 76, no. 2, pp. 301-314, 1994.

[2] M. S. Kluger, "Vascular endothelial cell adhesion and signaling during leukocyte recruitment," Advances in Dermatology, vol. 20, pp. 163-201, 2004.

[3] J. D. van Buul, F. P. J. Mul, C. E. van der Schoot, and P. L. Hordijk, "ICAM-3 activation modulates cell-cell contacts of human bone marrow endothelial cells," Journal of Vascular Research, vol. 41, no. 1, pp. 28-37, 2004.

[4] R. W. Tilghman and R. L. Hoover, "E-selectin and ICAM-1 are incorporated into detergent-insoluble membrane domains following clustering in endothelial cells," FEBS Letters, vol. 525 , no. $1-3$, pp. 83-87, 2002.

[5] A. Viola and N. Gupta, "Tether and trap: regulation of membrane-raft dynamics by actin-binding proteins," Nature Reviews Immunology, vol. 7, no. 11, pp. 889-896, 2007.

[6] J. D. van Buul, E. Kanters, and P. L. Hordijk, "Endothelial signaling by Ig-like cell adhesion molecules," Arteriosclerosis, Thrombosis, and Vascular Biology, vol. 27, no. 9, pp. 18701876, 2007.

[7] B. Wójciak-Stothard, L. Williams, and A. J. Ridley, "Monocyte adhesion and spreading on human endothelial cells is dependent on Rho-regulated receptor clustering," Journal of Cell Biology, vol. 145, no. 6, pp. 1293-1307, 1999.

[8] T. L. Deem, H. Abdala-Valencia, and J. M. Cook-Mills, "VCAM-1 activation of endothelial cell protein tyrosine phosphatase 1B," Journal of Immunology, vol. 178, no. 6, pp. 3865-3873, 2007.

[9] S. van Wetering, N. van den Berk, J. D. van Buul, et al., "VCAM-1-mediated Rac signaling controls endothelial 
cell-cell contacts and leukocyte transmigration," American Journal of Physiology, vol. 285, no. 2, pp. C343-C352, 2003.

[10] O. Barreiro, M. Yáñez-Mó, M. Sala-Valdés, et al., "Endothelial tetraspanin microdomains regulate leukocyte firm adhesion during extravasation," Blood, vol. 105, no. 7, pp. 2852-2861, 2005.

[11] J. D. van Buul, C. Voermans, V. van den Berg, et al., "Migration of human hematopoietic progenitor cells across bone marrow endothelium is regulated by vascular endothelial cadherin," Journal of Immunology, vol. 168, no. 2, pp. 588-596, 2002.

[12] O. Barreiro, M. Yáñez-Mó, J. M. Serrador, et al., "Dynamic interaction of VCAM-1 and ICAM-1 with moesin and ezrin in a novel endothelial docking structure for adherent leukocytes," Journal of Cell Biology, vol. 157, no. 7, pp. 1233-1245, 2002.

[13] P. L. Hordijk and J. D. van Buul, "Endothelial adapter proteins in leukocyte transmigration," Thrombosis and Haemostasis, vol. 101, no. 4, pp. 649-655, 2009.

[14] U. Cavallaro, J. Niedermeyer, M. Fuxa, and G. Christofori, "NCAM modulates tumour-cell adhesion to matrix by inducing FGF-receptor signalling," Nature Cell Biology, vol. 3, no. 7, pp. 650-657, 2001.

[15] L. Yang, J. R. Kowalski, P. Yacono, et al., "Endothelial cell cortactin coordinates intercellular adhesion molecule-1 clustering and actin cytoskeleton remodeling during polymorphonuclear leukocyte adhesion and transmigration," Journal of Immunology, vol. 177, no. 9, pp. 6440-6449, 2006.

[16] R. W. Tilghman and R. L. Hoover, "The Src-cortactin pathway is required for clustering of E-selectin and ICAM-1 in endothelial cells," The FASEB Journal, vol. 16, no. 10, pp. 1257$1259,2002$.

[17] M. J. Allingham, J. D. van Buul, and K. Burridge, "ICAM1-mediated, Src- and Pyk2-dependent vascular endothelial cadherin tyrosine phosphorylation is required for leukocyte transendothelial migration," Journal of Immunology, vol. 179, no. 6, pp. 4053-4064, 2007.

[18] O. Barreiro, M. Zamai, M. Yáñez-Mó, et al., "Endothelial adhesion receptors are recruited to adherent leukocytes by inclusion in preformed tetraspanin nanoplatforms," Journal of Cell Biology, vol. 183, no. 3, pp. 527-542, 2008.

[19] F. Berditchevski and E. Odintsova, "Tetraspanins as regulators of protein trafficking," Traffic, vol. 8, no. 2, pp. 89-96, 2007.

[20] M. E. Hemler, "Tetraspanin functions and associated microdomains," Nature Reviews Molecular Cell Biology, vol. 6, no. 10, pp. 801-811, 2005.

[21] M. Yáñez-Mó, M. Mittelbrunn, and F. Sánchez-Madrid, "Tetraspanins and intercellular interactions," Microcirculation, vol. 8, no. 3, pp. 153-168, 2001.

[22] S. Levy and T. Shoham, "The tetraspanin web modulates immune-signalling complexes," Nature Reviews Immunology, vol. 5, no. 2, pp. 136-148, 2005.

[23] S. Dijkstra, G. Kooij, R. Verbeek, et al., "Targeting the tetraspanin CD81 blocks monocyte transmigration and ameliorates EAE," Neurobiology of Disease, vol. 31, no. 3, pp. 413421, 2008. 

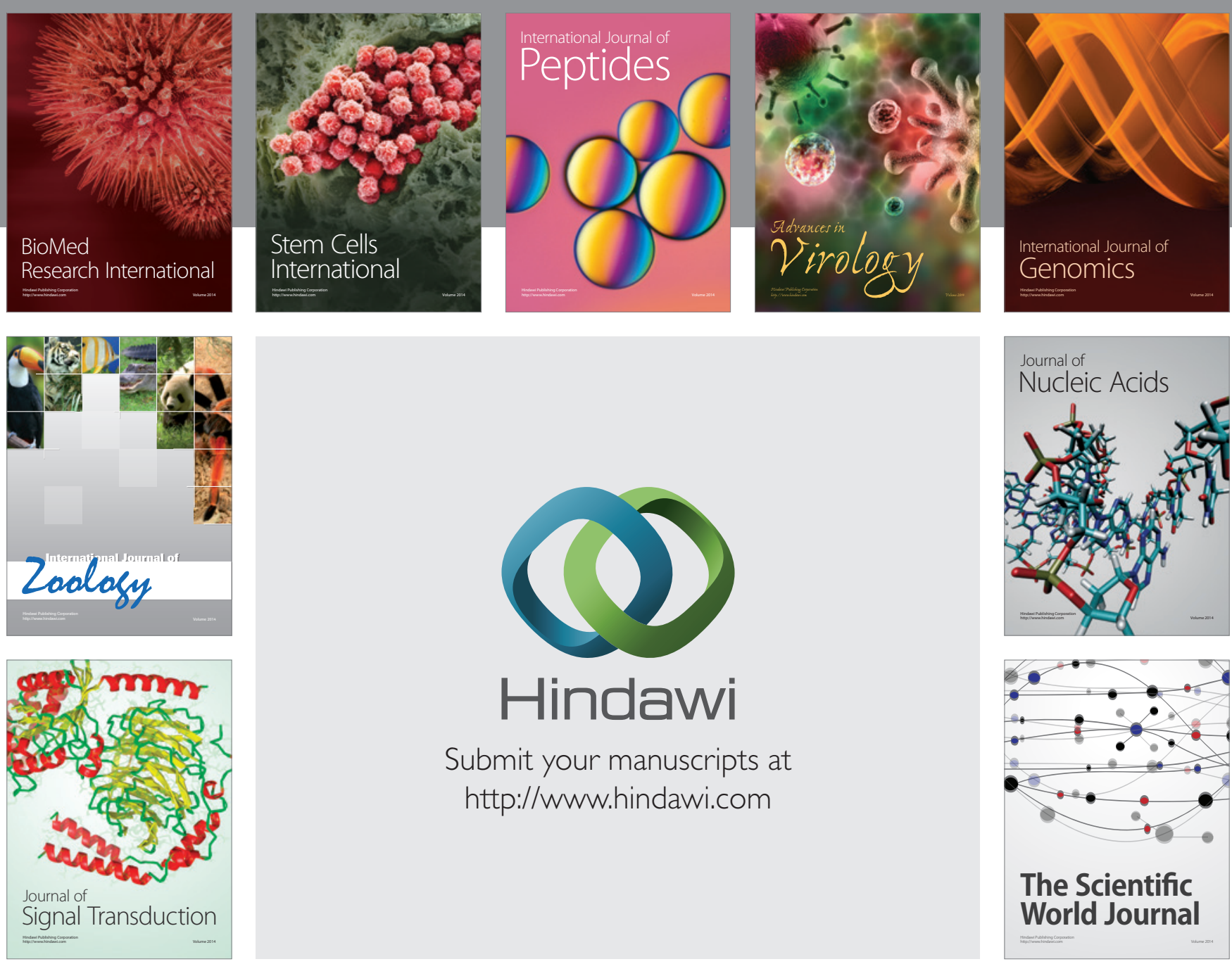

Submit your manuscripts at

http://www.hindawi.com
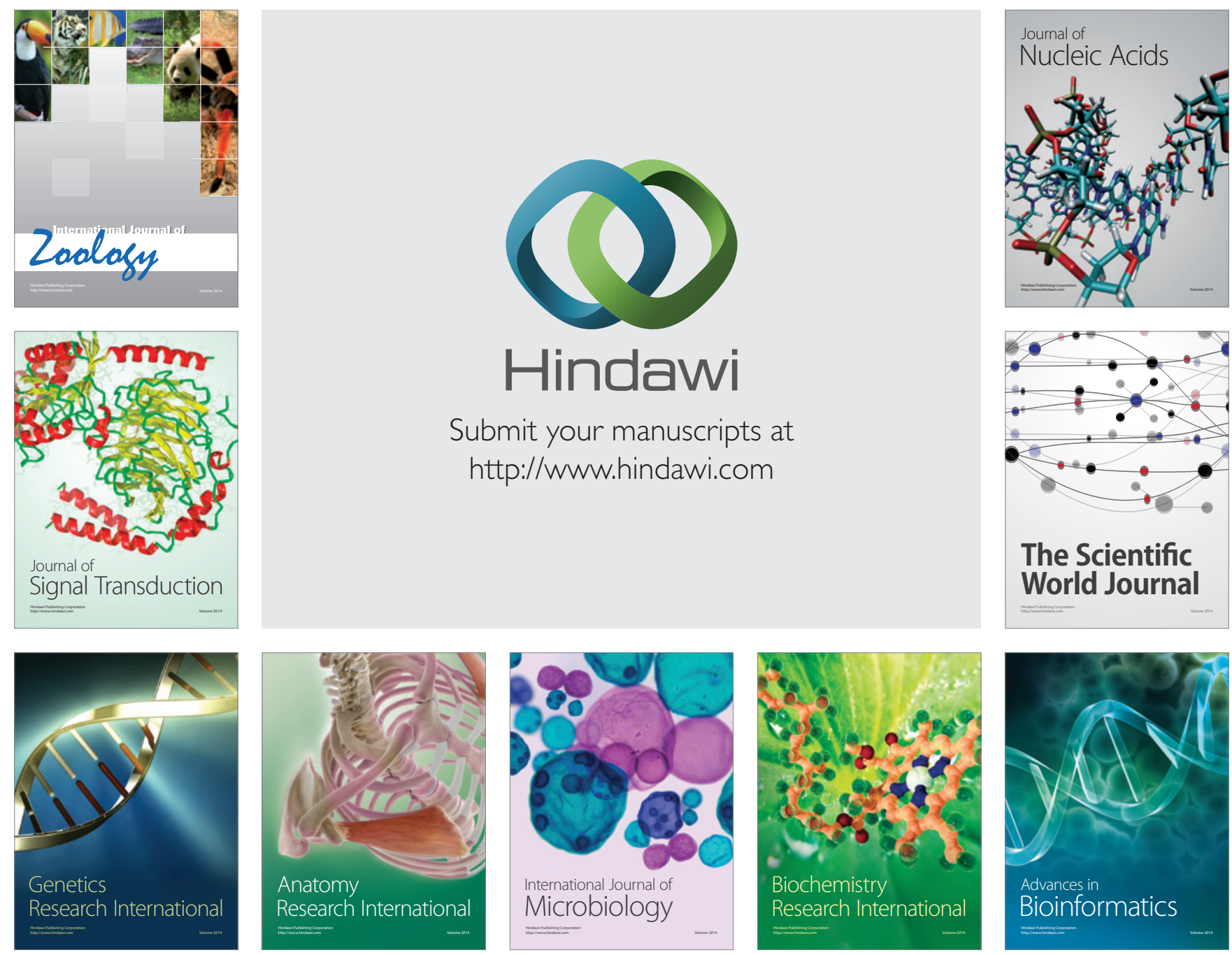

The Scientific World Journal
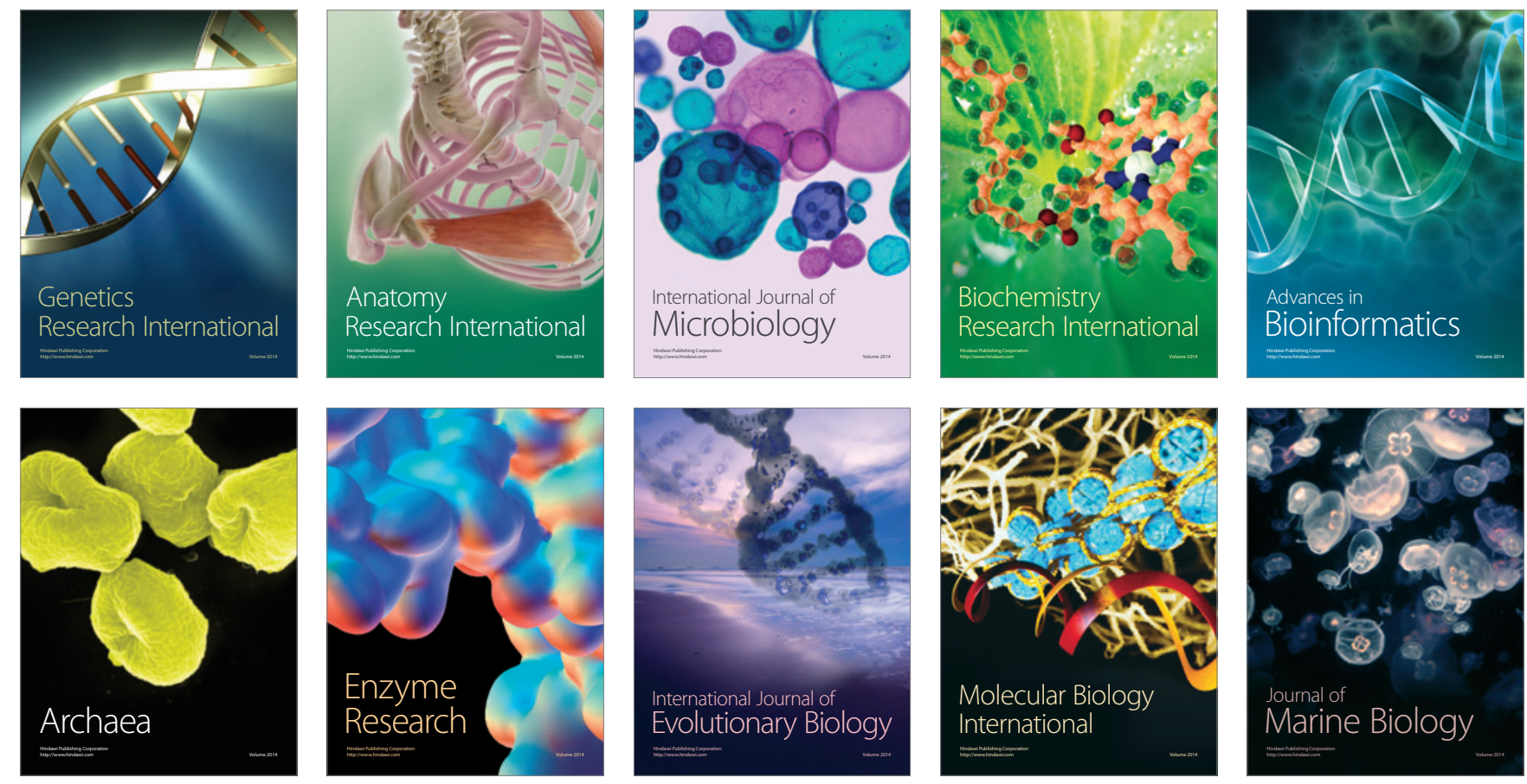